\title{
OLWRED: Best Selected Strategy for Data Transmission in Heterogeneous Networks
}

\author{
Wisam Mahmood \\ Lafta \\ School of Computer \\ Science, \\ Huazhong University of \\ Science and Technology, \\ Wuhan 430074, P.R. \\ China
}

\author{
Saba Qasim Jabbar \\ School of Electronics \& \\ Information Engineering \\ Huazhong University of \\ Science and Technology \\ Wuhan 430074, P.R. \\ China
}

\author{
Dheyaa Jasim \\ Kadhim \\ Electrical Engineering \\ Department, \\ College of Engineering, \\ University of Baghdad \\ 47024, \\ Baghdad, Iraq
}

\author{
Guangzhi Ma \\ School of Computer \\ Science, Huazhong \\ University of Science \\ and Technology, \\ Wuhan 430074, P.R. \\ China
}

\begin{abstract}
The routing protocols are used for wireless networks, cannot be used for wired networks because of the network's mobility. A varying of network conditions with variety of routing protocols are analyzed to find an optimized path from a source to destination. Use the appropriate protocol strengthens the data transfer process and it is very necessary to ensure the speed of the flow of data traffic and to avoid the congestion due to a bottleneck in the network. There is a common major problem in wired and wireless networks (heterogeneous network) namely a congestion, which affects the speed and accuracy access packages. Congestion is the biggest challenge that appears when the transmission of data from the source to the destination and it can be measured by several parameters such as; network throughput, utilization of network and the queue delay. This paper proposed a new strategy representing the fast and suitable approach to transmit a huge data according to weight random early detection (WRED) strategy with different kind of wireless routing protocols (reactive routing protocols, proactive routing protocols and hybrid routing protocols), to get the best performance with heterogeneous network. The network performance is simulated using OPNET simulation software.
\end{abstract}

\section{General Terms}

Heterogeneous networks, Wireless Networks, Routing Protocols, OPNET.

\section{Keywords}

Heterogeneous networks, Routing, Congestion, WRED, OPNET.

\section{INTRODUCTION}

Wireless Ad-hoc networks uses different routing protocols, which can be categories in three types: (I) reactive (II) proactive and (III) hybrid [1][2]. The famous one of reactive routing protocols is dynamic source routing protocol (DSR), while the famous one of proactive routing protocols is optimized link state routing (OLSR), and the famous hybrid routing protocol is temporary ordered routing algorithm (TORA) [1][3]. To distinguish among these protocols, they can be also categories in two routing approaches; (I) congestion-control routing, and (II) congestion-non control routing, depending on the amount of data transferred between the source and destination [1][2][3]. To find out the link path in advance, the protocols mentioned above are classified as congestion controlled routing protocols [3][4].

The Congestion must be controlled in the modern communications networks such as Internet in order to avoid the data loss during transmission of packets between nodes, every day online transmits a large amount of data which it is easy to show the problem of congestion. Now, to avoid the problem of congestion, it must be enabled active queue management (AQM) by using weight random early detection (WRED) strategy [4][5][6]. The problem of congestion appears clearly when the number of packets arriving to the queue more than the number of packets Departure [4][7]. There are several general reasons that cause network problems which they must be addressed in addition to the congestion, and these problems are shared in various types of networks, these reasons are listed below:

- Bandwidth constraint is limited.

- Buffer space of intermediate nodes is limited

- Multipath routing procedures not active.

- Efficient load balancing means that equally partition load at each node on the network properly.

- Unsteady packet submission, end to end lateness and packet losses in case of link failure.

This paper is organized as follows: summarization of the strategies appears in section 2. Section 3 presents the proposed work. The simulation environments and results are presented in section 4. Finally, the conclusions of this paper are shown in section 5 .

\section{SYSTEM MODEL}

The speed and accuracy of transmitted packet are critical issues in heterogeneous networks with avoiding traffic drop and congestion. The main problem for the regression of quality of service (QoS) in the Internet is congestion, the congestion appears in TCP protocol when a packet has been dropped at the gateway, since TCP doesn't work with high harmonic wireless environment because of the mobile environment's nature and it is designed to work with wired environment [1][4][8]. TCP's strategy work is to control on the congestion, as opposed in trying to avoid congestion in the first place. TCP often increases the load as it schedules on the network in trying to find the point at which congestion occurs, and then it decides to bypass this point [8][9].

So, this paper proposed a dynamic queue and multipath based routing strategies for heterogeneous networks which they are more suitable for work with TCP to control the congestion and manage the memory requirements as compared to existing queue based congestion control [8][10]. In addition to use the WRED strategy with different routing protocols and applying 
this proposed approach in gateway (routers) to select the best strategies in the simulation as shown in Fig. 1 below. The proposed is trying to the best results based on several criteria with specific purpose of the application in order to control or avoid the congestion by reducing delays in the queue and increase the throughput and utilization of heterogeneous network.

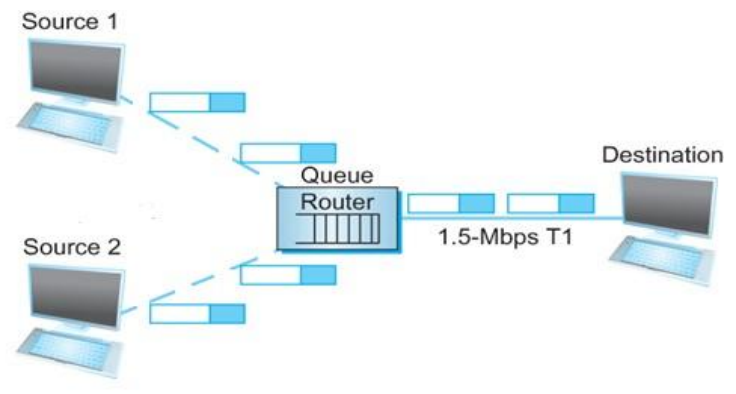

Fig 1: Proposed System Model

\subsection{WRED/RED Strategies}

The WRED/RED strategies are representative algorithms of Active Queue Management (AQM) [9], and they are used for congestion avoidance based on FIFO queue system priority policy [10]. The basic idea of FIFO queuing which is also called first-come-first-served (FCFS) queuing, the first packet that arrives in the buffer of queue at router is the first packet to be transmitted because of the amount of buffer space at each router is limited, if the queue (buffer space) is full when the new packet arrives, then the router neglects this packet. This is sometimes named Tail Drop [10][11], previous packets that arrive at the tail end of the FIFO are dropped. That tail drop and FIFO are two separable different work ideas. FIFO is a scheduling discipline that organizes choosing the order in which packets are transmitted. Tail drop is a drop policy - it selects which packets get dropped [11-14], Fig. 2 below shows the principle concept of tail drop. The FIFO mechanism cannot send information to alert to the TCP protocol only after the occurrence of congestion, when queue overflow is happened since that the TCP makes many connections at the same time into slow start and this causes the occurrence of tail drop. The traffic restriction process can be ineffective in the long term to the fact that this method does not resolve the problem of congestion effectively, this phenomenon will claim to fluctuation of utilization and throughput of network link congestion due to the return time[8][13][14].

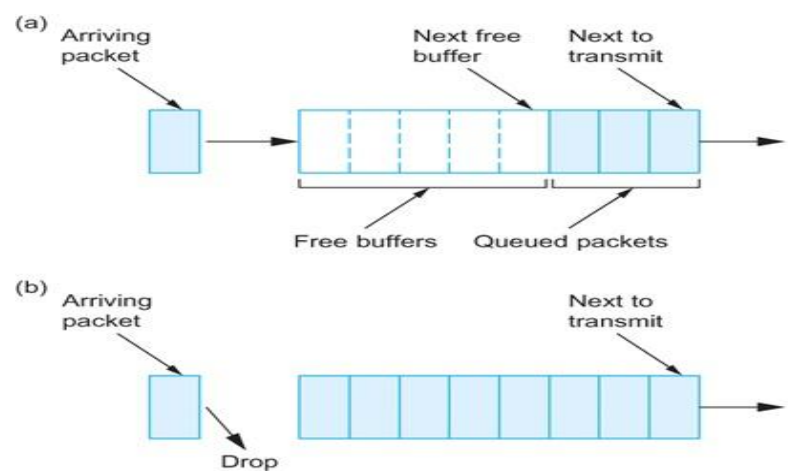

Fig 2: (a) FIFO queuing (b) Tail drop at a FIFO queue.

The RED is design and programmed to be used in conjunction with TCP by monitor the router queue length and when it detects that congestion is imminent Source learn to repair congestion window [8][14][15]. In other words, the router drops a few packets before overflow buffer space completely, in this case should the source slow down [11][13][15].

Firstly, RED calculates an average queue length using a weighted functionality average similar to the one used in the premier TCP timeout counting as shown in Fig. 3 below, then Avger $_{l}$ is given by

Avger $_{1}=(1-$ Weight $) \times$ Avger $_{1}+$ Weight $\times$ SampleLen

Secondly, RED computation will be done using two queue length thresholds that trigger certain activity; minimum threshold $\left(\mathrm{MIN}_{\mathrm{th}}\right)$ and maximum threshold $\left(\mathrm{MAX}_{\mathrm{th}}\right)$ according to the following rules:

- If Avger $_{1} \leq M I N_{t h} \rightarrow$ queue the packet

- If $M I N_{t h}<$ Avger $_{l}<M A X_{t h} \rightarrow$ compute probability $\mathrm{P}_{\mathrm{L}} \rightarrow$ drop the arriving packet with probability $\mathrm{P}_{\mathrm{L}}$

- if $M A X_{t h} \leq$ Avger $_{l} \rightarrow$ drop the arriving packet

- $\quad P_{L}$ is a function of both Avger ${ }_{1}$ and how long it has been ago the last packet was dropped.

- Specifically, it is calculated as follows:

$\operatorname{TempP}_{L}=\operatorname{MaxP}_{L} \times\left(\right.$ Avger $\left._{1}-\operatorname{MIN}_{t h}\right) /\left(M A X_{t h}-M I N_{t h}\right)$

$P L=\operatorname{Temp}_{L} /\left(1-\right.$ count $\left.\times \operatorname{Temp}_{L}\right)$

Where $0<$ Weight $<1$ and SampleLen is the length of the queue when a sample measurement is made.

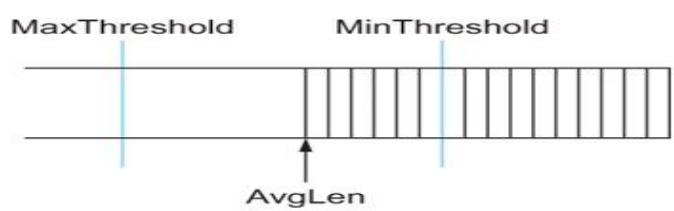

Fig 3: RED thresholds at FIFO queue

In WRED strategy implementation, the queue length is measured every time as a new packet arrives at the gateway, however, this is the main difference between RED and WRED strategies. Calculating the average of queue length periodically, gives WRED strategy more robust and makes the queue more sensitive to excess data transfer as shown in Fig.4 below.

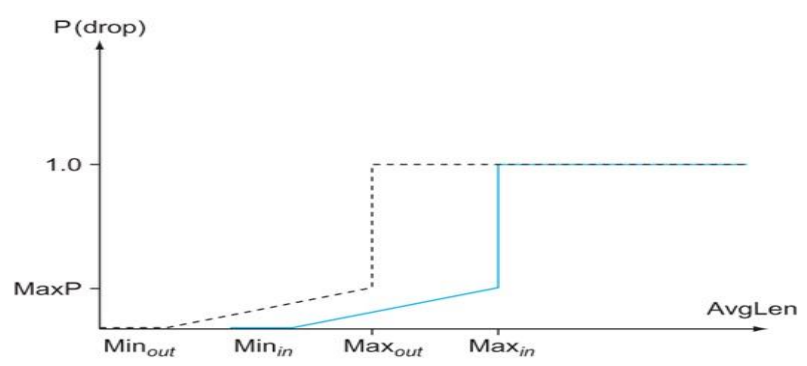

Fig 4: WRED strategy implementation

\subsection{Routing Protocols}

There are three categories of routing protocols in wireless networks that they are well known such as reactive, proactive and hybrid routing protocols. In this work, It will consider all these three types of routing in order to choose the best one of these routing protocols to work with the WRED and RED strategies, so the performance of the network can be enhanced to solve the problem of congestion with depending on using $\mathrm{TCP}$ protocol in upper transport layer. 
The reactive routing protocol is called also on demand routing protocol. It works when the source start sends data to the specific destination and then the route discovery mechanism is initiated. The route to the destination node is thus maintained and it remains so till the time when the destination becomes unreachable or the route becomes invalid. Dynamic Source Routing Protocol (DSR) is the example of Reactive routing protocols [1][16]. The proactive routing protocol is called also table driven routing, which it works by maintaining the fresh list of destinations and their routes by periodically deployed routing tables throughout the network. Optimized link state routing protocol (OLSR) is the example of Proactive routing protocols [17][18]. In other side, the hybrid routing protocol contains the traits of both the above mentioned routing protocols. Temporary Ordered Routing Algorithm (TORA) is the example of the hybrid routing protocols. TORA attempts to achieve a high degree of scalability by using a nonhierarchical routing algorithm. In this situation the algorithm attempts to crush, to the greatest rang possible, the TORA does not use a shortest path link. TORA It contains a table of the tracks and maintains a linked Acyclic Graph rooted at a destination [18][19]. The key main concepts of TORA are localization of control messages to a so few collection of nodes near the manifestation of a topological change. To finish this, nodes need to maintain the routing Information about the neighboring node.

\subsection{Compatibility between TCP and OLSR}

Through the studying to TCP protocol and above routing protocol, it can notice the big similarity and compatibility between TCP and OLSR because of their seeking to provide the best and reliable link through the networks. TCP is a connection-oriented allowing raw information exchange protocol. It runs a mechanism to establish the connection, which it is the designated 3-Way Handshake. During these three exchanges, the sender and receiver agree on the size of the buffers to be used for sending and receiving TCP segments exchanged the maximum size of a frame of an Ethernet network call MTU (Maximum Transfer Unit). TCP also some acknowledgments (ACKs) are executed to allow progressively validate received frames [1][3][19].

However, OLSR is a proactive link-state routing protocol work is divided into two phases: Through first phase, it uses Topology Control (TC) messages and Hello to discover and then bring out link state information throughout the network. Individual nodes use this topology information to set next hop nodes for all nodes in the network using shortest path. Using Hello messages and TC, the OLSR protocol at each node sets two hops neighbor information and performs a distributed election of a set [3][7][17][18]. While at the second phase, it begins working when the each of its two hops neighbors send answer (acknowledgment) to the source node. Multipoint distribution relays (MPRs) uses nodes of sources and directions of TC messages that keep the MPR selectors paths. This processing of MPRs makes OLSR distinct from other link state routing protocols in a few different routine: The progressing path for TC messages is not participate among all nodes but varies according to the source, only a part of nodes source connect state information, not all connects of a node are decelerated but only those that represent MPR selections [1][2][14][17][19].

\section{SIMULATION RESULTS}

The simulation environment is built from heterogeneous network in area $2500 \mathrm{~m} \times 5000 \mathrm{~m}$, which contains two environments (wired and wireless). The wired environment consists from two routers, server (source), switch and access point and all these are connected by each other through wire links. While the wireless environments are interconnected 24 nodes (destinations) randomly deployed and connected through access point, and they are working by order demand. The devices should be set with duration $=600$ seconds to transfer data of (60 Gbit/second) and (500 packet/ second), also to transfer FTP file on demand with type of service best effort as shown in Fig. 5 below.

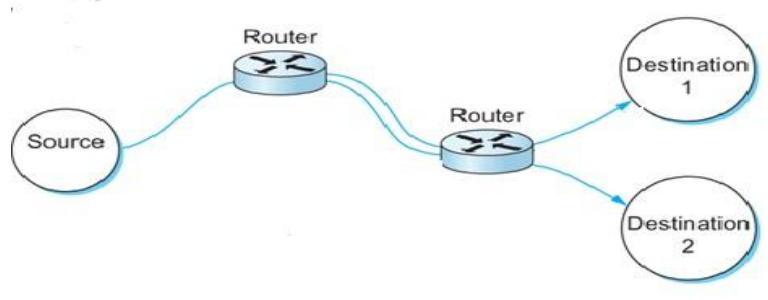

Fig 5: Simulation Environment

Then, it implemented this heterogeneous network for the suggested simulation scenarios listed in Table 1 below, so it can get the simulation results as shown in the following figures below for the most important performance parameters (queue delay, throughput, and utilization). The following points are described these parameters:

- Queue delay: It is the job time which waits in a queue until it can execute, this term is almost used in reference to routers. Also it can be defined as the time spending in the router to be processed and transmitted.

- Throughput: This term is used to measure the performance of a network, it is the number of packets successfully delivered per unit time and controlled on available bandwidth.

- Utilization: This term is used to host a larger amount of traffic on the different networks, thus reducing operation cost and being the foundation for hosting the exponential growth of modern networks.

Table 1. Simulation Scenarios

\begin{tabular}{|c|c|c|c|c|c|c|c|}
\hline & $\begin{array}{c}\text { Queue } \\
\text { pro. }\end{array}$ & $\begin{array}{c}\text { Max } \\
\text { queue } \\
\text { size }\end{array}$ & Wgt & MIN $_{\text {th }}$ & MAX $_{\text {th }}$ & Exp & $\begin{array}{c}\text { Packet } \\
\text { size } \\
\text { (BPS) }\end{array}$ \\
\hline $\begin{array}{c}\text { Scenario } \\
1 \\
\begin{array}{c}\text { Without } \\
\text { any } \\
\text { strategy }\end{array}\end{array}$ & FIFO & 85 & 9 & 100 & 200 & 0.05 & 500000 \\
\hline $\begin{array}{c}\text { Scenario } \\
2 \\
\text { OLSR } \\
\text { with } \\
\text { WRED }\end{array}$ & FIFO & 85 & 12 & 5 & 15 & 0.05 & 500000 \\
\hline $\begin{array}{c}\text { Scenario } \\
3 \\
\text { TORA } \\
\text { with } \\
\text { WRED }\end{array}$ & FIFO & 85 & 12 & 5 & 15 & 0.05 & 500000 \\
\hline $\begin{array}{c}\text { Scenario } \\
4 \\
\text { DSR } \\
\text { with } \\
\text { WRED }\end{array}$ & FIFO & 85 & 12 & 5 & 15 & 0.05 & 500000 \\
\hline $\begin{array}{c}\text { Scenario } \\
5 \\
\text { Only } \\
\text { WRED }\end{array}$ & FIFO & 85 & 12 & 5 & 15 & 0.05 & 500000 \\
\hline
\end{tabular}

Through Fig. 6 below, it can note the presence of five scenarios built together in order to get the average packet delay rate in the queue for first different strategies such as (WRED only, OLSR with WRED, TORA with WRED, and DSR with WRED). Fig. 6 shows that there is an improvement of using WRED over no using any strategy, and there is 
another improvement of using WRED with routing protocol over using just WRED strategy. In another side, it show from this figure that there is no difference of using different routing protocols with WRED, which they have the approximately equal queuing delays.

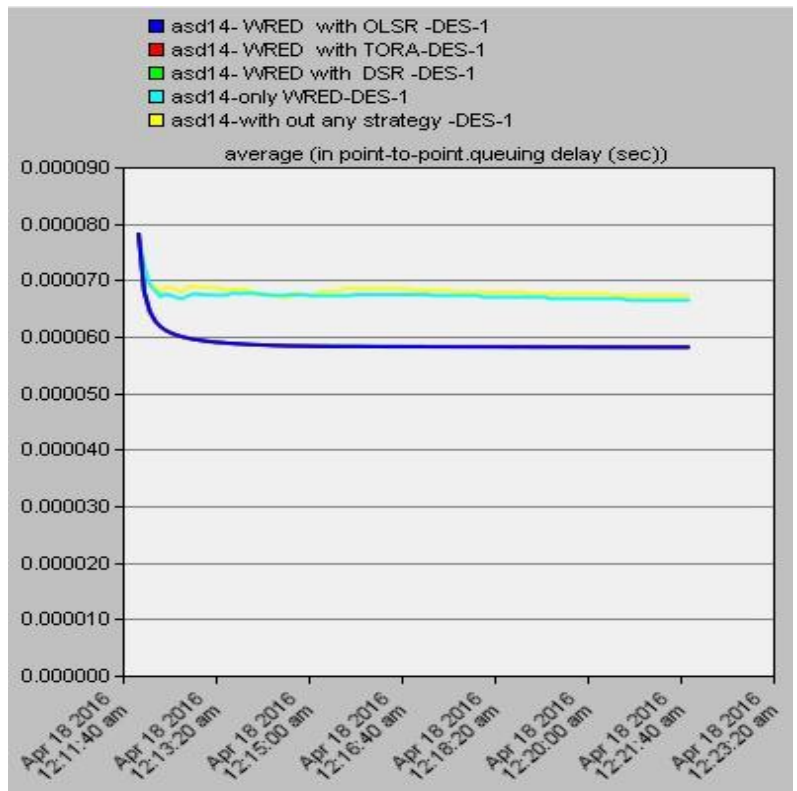

Fig 6: Average queuing delay for different scenarios

Fig. 7 shows the average throughput for the five different scenarios with considering the same environment and with the same circumstance. If it compares the rest of the curves with each other, it concludes that the best productivity is represented in the WRED with OLSR, then TORA, then DSR, then only WRED and then without applying any strategy respectively.

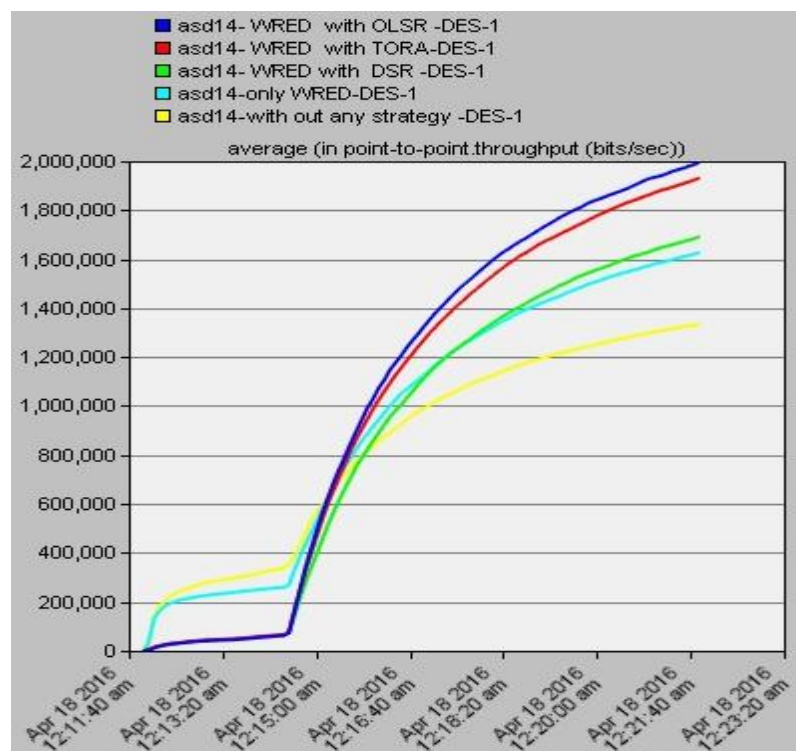

Fig 7: Average throughput for different scenarios

Fig. 8 shows that the best performance among the five different strategies WRED with OLSR since it gives the better network utilization rather than other strategies, following by TORA with WRED, DSR with WRED, WRED only and without apply any strategy respectively.

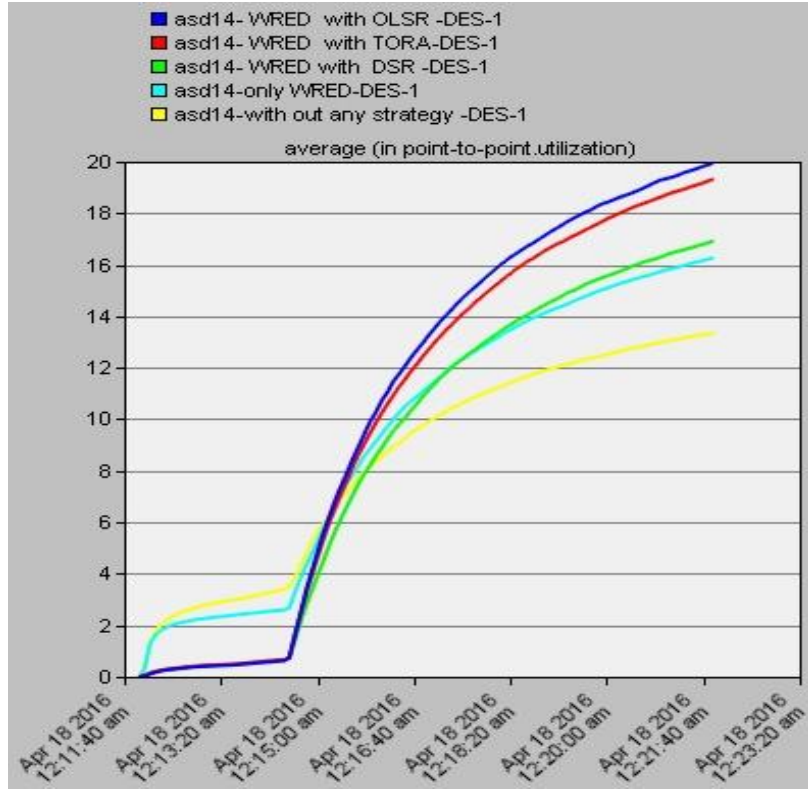

Fig 8: Average Utilization for different scenarios

\section{CONCLUSIONS}

It can conclude from the simulation results are described in the previous section, and through applying of different routing protocols with the WRED strategy in heterogeneous network. It note that the best strategy is WRED with the OLSR since they were able to scale down the delay in queue with a clear increasing of the network performance and productivity, and also scoffed at all the best possibility for the use of network resources through high utilization. So it has a new proposed strategy which it is a compacted from OLSR with WRED, and it called it as (OLWRED). This new strategy can avoid the congestion by reducing the queue delay and increasing the performance of the heterogeneous networks. The future work can programing this strategy in node itself (specific nod) not on all the network.

\section{REFERENCES}

[1] Kumaran, Senthil, and V. Sankaranarayanan. "Congestion Free Routing in Ad-hoc Networks." Issue 6, ISSN. 2012

[2] Ramanathan, R. and J. Redi, 2002. "A brief overview of ad hoc networks: Challenges and directions". IEEE Commun. Mag., 40: 20-22. DOI

[3] Mughal, Fouzan Zulfiqar, and Mohammad Imran Azam. "Comparative analysis of proactive, reactive and hybrid ad hoc routing protocols in Client based Wireless Mesh Network." Information and Emerging Technologies (ICIET), 2010 International Conference on. IEEE, 2010.

[4] Adams, Richelle. "Active queue management: a survey." IEEE communications surveys \& tutorials 15.3 (2013): 1425-1476.

[5] Lafta, Wisam Mahmood, Saba Qasim Jabbar, and Guangzhi Ma. "Performance Evaluation of Heterogeneous Network Based on RED and WRED." Indonesian Journal of Electrical Engineering and Computer Science3.3 (2016): 540-545

[6] Yadav, Manoj, Sachin Kumar Gupta, and R. K. Saket. "Multi-hop wireless ad-hoc network routing protocols-a comparative study of DSDV, TORA, DSR and 
AODV." Electrical, Electronics, Signals, Communication and Optimization (EESCO), 2015 International Conference on. IEEE, 2015.

[7] $\mathrm{Vu}, \mathrm{T}$. "Effect of RED and different packet sizes on Multimedia performance over wireless networks." TU Ilmenau, Germany (2000): 1-7.

[8] Hollot, Chris V., et al. "On designing improved controllers for AQM routers supporting TCP flows." INFOCOM 2001. Twentieth Annual Joint Conference of the IEEE Computer and Communications Societies. Proceedings. IEEE. Vol. 3. IEEE, 2001

[9] Gupta, Hitesh, and Pankaj Pandey. "Congestion Control Using Varying Queue Base Approach as Well as Multipath Routing Under MANET."International Journal of Engineering Research and Technology. Vol. 2. No. 12 (December-2013). ESRSA Publications, 2013.

[10] Zhi-bo, C. A. O. "Simulation and Optimization of RED Algorithm [J]."Computer Technology and Development 8 (2010): 188-191.

[11] Gupta, Dinesh, and Deepak Singh Tomar. "Dynamic Queue and TCP based Multipath Congestion Control Scheme for Wired Network." International Journal of Computer Applications 123.10 (2015).

[12] Braden, Bob, et al. "Recommendations on queue management and congestion avoidance in the Internet". No. RFC 2309. 1998
[13] Jiang, Rui-juan, et al. "Simulation Study of RED/WRED Mechanism Based on OPNET." (2015).

[14] Mishra, Shweta, and Nidhi Jain. "Effective Impact of ECN with RED to Control Heavy Congestion."

[15] Floyd, Sally, and Van Jacobson. "Random early detection gateways for congestion avoidance." IEEE/ACM Transactions on networking 1.4 (1993): 397-413.

[16] Jacobson, Van. "Congestion avoidance and control." ACM SIGCOMM computer communication review. Vol. 18. No. 4. ACM, 1988.

[17] Sarkar, Priyanka, and Hrituparna Paul. "Comparative performance analysis of GZRP, AOMDV and DSR in MANETs." Computational Intelligence and Information Technology, 2013. CIIT 2013. Third International Conference on. IET, 2013.

[18] Kassim, Murizah, Ruhani Ab Rahman, and Roihan Mustapha. "Mobile ad hoc network (MANET) routing protocols comparison for wireless sensor network."System Engineering and Technology (ICSET), 2011 IEEE International Conference on. IEEE, 2011.

[19] Fan, Xunli, et al. "Qblue: A new congestion control algorithm based on queuing theory." High Performance Computing and Communication \& 2012 IEEE 9th International Conference on Embedded Software and Systems (HPCC-ICESS), 2012 IEEE 14th International Conference on. IEEE, 2012. 